\title{
Protein structures as shapes: Analysing protein structure variation using geometric morphometrics
}

\author{
Jose Sergio Hleap \\ Department of Human Genetics \\ McGill University \\ Canada \\ Christian Blouin \\ Faculty of Computer Science \\ Dalhousie Univeristy \\ Canada
}

November 13, 2017

\begin{abstract}
A phenotype is defined as an organism's physical traits. In the macroscopic world, an animal's shape is a phenotype. Geometric morphometrics (GM) can be used to analyze its shape. Let's pose protein structures as microscopic three dimensional shapes, and apply principles of GM to the analysis of macromolecules. In this paper we introduce a way to 1) abstract a structure as a shape; 2) align the shapes; and 3) perform statistical analysis to establish patterns of variation in the datasets. We show that general procrustes superimposition (GPS) can be replaced by multiple structure alignment without changing the outcome of the test. We also show that estimating the deformation of the shape (structure) can be informative to analyze relative residue variations. Finally, we show an application of GM for two protein structure datasets: 1) in the $\alpha$-amylase dataset we demonstrate the relationship between structure, function, and how the
\end{abstract}


dependency of chloride has an important effect on the structure; and 2) in the Niemann-Pick disease, type C1 (NPC1) protein's molecular dynamic simulation dataset, we introduce a simple way to analyze the trajectory of the simulation by means of protein structure variation.

Keywords: Procrustes superimposition, Shape analysis, Protein structure comparison

\section{Introduction}

Geometric morphometrics is a collection of approaches for the multivariate statistical analysis of Cartesian coordinate data [Slice, 2007]. The "geometry" referred to by the word "geometric" is the estimation of mean shapes and the description of sample variation of shape using the Procrustes distance (Kendall's shape space) [Rohlf, 2002]. It is mainly based on landmarks which are "discrete anatomic loci that can be recognized as the same loci in all specimens of study" [Zelditch et al., 2004, p.443], and must: be homologous anatomical loci, not alter their topology or position relative to other landmarks, provide adequate coverage of the morphology, be consistently assigned, and lie within the same plane [Zelditch et al., 2004].

Landmark data is more informative than traditional data (linear measurements in the geometric morphometrics context) since its coordinates also contain positional information and thus geometric structure. Once homologous landmarks are assigned, "noisy" factors affecting the dimensionality and degrees of freedom of the possible shape analysis are removed by means of generalized Procrustes superimposition (GPS). Such factors being rotation, translation and size, and are dealt with by [Adams et al., 2004, Zelditch et al., 2004]:

1. Assign homologous landmarks to meaningful and descriptive parts of the shape.

2. Center each configuration of landmarks at the origin by subtracting the coordinates of its centroid from the corresponding ( $\mathrm{X}$ or $\mathrm{Y}$ ) coordinates of each landmark: Removing positional variation by translating each centroid to the origin.

3. Scale the landmark configuration to unit centroid size by dividing each coordinate of each landmark by the centroid size of the configuration. 
4. Set one configuration as a reference and rotate the other configurations to minimize the summed squared distances between homolog landmarks, thus removing rotational variation.

The above method can be expressed as [Rohlf and Slice, 1990]:

$$
X_{i}=\rho X H+1 \tau
$$

where matrix $X$ is the original configuration; $\rho$ is the scaling done to $X ; 1 \tau$ is the translation performed to $X_{i}$ to a reference position; and $H$ is a rotation (with an angle of rotation $\theta$ ) matrix of the form:

$$
H=\left[\begin{array}{cc}
\cos \theta & -\sin \theta \\
\sin \theta & \cos \theta
\end{array}\right]
$$

Then, the set of all matrices representing the landmark configurations (configuration space), becomes the shape space, and its dimensions are given by:

$$
K M-(M+1)
$$

where $K$ is the number of landmarks, and $M$ the number of dimensions in each landmark. After removing the effects of size, rotation and translation, $2 K-4$ degrees of freedom are left for $2 \mathrm{D}$ data and $3 K-7$ for $3 \mathrm{D}$ data [Zelditch et al., 2004].

The comparison and analysis of the superimposition is based in the the Procrustes distance $\left(D_{P}\right)$. GPA applies the Procrustes analysis method to align a population of shapes instead of only two shape instances [Dryden and Mardia, 1998]. The Procrustes distance is the square root of the sum of squared differences between the positions of the landmarks in two optimally superimposed configurations $\left(C_{1}\right.$ and $\left.C_{2}\right)$ [Rohlf, 2002]:

$$
d_{P}^{2}=\sum_{j=1}^{K}\left[\left(x_{j 1}-x_{j 2}\right)^{2}+\left(y_{j 1}-y_{j 2}\right)^{2}\right]
$$

where $d_{P}^{2}$ is the squared Procrustes distance, and $K$ is the number of landmarks. This metric can then be used in several statistical multivariate analyses to attain differences in shapes, clustering, changes in time, test symmetry, etc. Here shapes can be treated as a single point in a multidimensional space, and therefore the information can be summarized in an efficient way using standard multivariate techniques. Some traits can also be treated 
independently in the analyses, extracting information of particular aspects of the shape.

This paper shows how these geometric morphometric methods and applications can be use to analyze protein structure variation. It will start by proposing a method to abstract a protein structure as a shape. Then a demonstration that the scaling applied in the General Procrustes Superimposition (GPS), which does not apply to protein structures, can be omitted without changing the results will be shown. It will also be shown that GPS can be replaced by a Multiple Structural Alignment (MStA), which handles the rotation and translation part of the transformation. A simple method to analyze individual residue variation within this context and relate it to structural issues will be introduced. Finally, we will show general applications of GM-like methods for protein structure variation analysis in two datasets of actual protein structures.

\section{Material and methods}

\section{Abstracting a protein structure as a shape}

A landmark will be defined as the centroid of each homologous residue, and is defined by $(x, y, z)$ :

$$
\left(\frac{1}{A} \sum_{j=1}^{A} X_{j}, \frac{1}{A} \sum_{j=1}^{A} Y_{j}, \frac{1}{A} \sum_{j=1}^{A} Z_{j}\right)
$$

where $A$ will be the number of heavy atoms that constitutes the side chain of a residue including the $C_{\alpha}$. This procedure takes into account only homologous residues assigned by a structural alignment using the MStA application MATT [Menke et al., 2008]. Only ungapped homologous sites will be taken into account.

\section{Testing the effect of scaling in protein datasets}

The HOMSTRAD (386 datasets) and SABmark (425 datasets) superfamily subsets reported in MATT's paper [Menke et al., 2008] were used. The former database was design to store structures based on the quality of the X-ray analysis and accuracy of the structure, while the latter database was designed 
to test multiple alignment problems. Residue homology was determined by MATT-reported alignment [Menke et al., 2008, http://groups.csail.mit. $\mathrm{edu} / \mathrm{cb} / \mathrm{matt} /$. The centroid's coordinates (see section Abstracting a protein structure as a shape) for each homologous residue were computed and stored. To the resulting centroid's coordinates file, GPS with and without scaling were performed with the $\mathrm{R}[\mathrm{R}, 2011$ ] package "Shapes" [Dryden, 2011]. A correlation analysis was performed graphically and using the Pearson test for correlation using the MatPlotLib [Hunter, 2007], and Scipy [Jones et al., 2001] libraries in Python.

\section{Testing GPS versus structural alignments in protein datasets}

As in section Testing the effect of scaling in protein datasets, the HOMSTRAD and SABmark datasets where used to test the effect of aligning the protein structures with GPS or the flexible MStA implementation in MATT. The matrix of pairwise RMSD per dataset were computed, and a correlation analysis was made following the methods of section Testing the effect of scaling in protein datasets.

\section{Analysis of the deformation in superimposed structures/shapes}

The inter-landmark distance matrix (form configuration) is computed using the Euclidean distance for each entry in $m$ dimensions:

$$
d(a, b)=\sqrt{\sum_{1}^{m}\left(a_{m}-b_{m}\right)^{2}}
$$

where $d(a, b)$ stands for the Euclidean distance between variables $a$ and $b$. Therefore the form matrix $(F M)$ is [Claude, 2008]:

$$
F M=\left(\begin{array}{ccc}
d_{1,1} & \cdots & d_{i, 1} \\
\vdots & \ddots & \vdots \\
d_{1, j} & \cdots & d_{i, j}
\end{array}\right)
$$

$F M$ is then a square symmetric matrix, with zeros in the diagonal entries. If two forms (shapes in the inter-landmark framework) are identical, they will have the same entries in the FM matrix. The matrix of differences in form 
[The form difference matrix or FDM as named by Claude, 2008] between two configurations $S 1$ and $S 2$ is given by:

$$
F D M_{\frac{S 1}{S 2}}=\frac{F M_{S 1}}{F M_{S 2}}
$$

The score of the most influential point $(I)$ in the data can be computed by adding the sum of the differences to the median value per column (variable) and ranking the positions. This can be computed as:

$$
I \leftarrow \max \left(\sum_{i=1}^{c}|F D M-\operatorname{median}(F D M)|_{i} .\right)
$$

where $c$ is the column index, and FDM is the form difference matrix.

However, as shown in equation 6, this FDM is the representation of the difference between two shapes. We can generalize by summing the residuals of all shapes versus a hypothetical mean shape. For simplicity this can be calculated as the per-variable per-dimension average. In other words, the average of each dimension of each landmark. This approach will then return a Form Difference (FD) value per landmark, however; this value is not bounded and is difficult to interpret. For this reason we scaled the resulting FD vector $(\overrightarrow{F D})$ such that it is bounded from -1 (least variation) to 1 (highest variation) with:

$$
F D_{s}=\left(\frac{\overrightarrow{F D}-\min (\overrightarrow{F D})}{\max (\overrightarrow{F D})-\min (\overrightarrow{F D})} * 2\right)-1
$$

To illustrate how this works, a simulation of 500 hexagons was performed. Giving an initial shape, for each point and each dimension in the point, a distribution of random normal numbers is created. This distribution has a given standard deviation (0.05 for regular points, 0.005 for low variation points, and 0.2 for highly variable points). The mean was set to 0 . This distribution per point and per dimension was then added to the original shape, creating the simulated dataset with controlled variation. To explore a more complex system than the hexagon, a protein simulation was also performed in the same fashion as for the $2 \mathrm{D}$ case. In this case more extreme points were used for visualization purposes, being 0.0005 the lowest variation and 0.5 the highest. The protein used for the simulation and visualization was the Porcine Pancreatic Amylase (PPA) with pdb code 1PPI.

To test the variation in an evolutionary perspective, a protein structure sampling was performed. A PFAM [Finn et al., 2010] seed alignment of the 
$\alpha$-Amylase family was gathered and used to seed a PSI-BLAST [Altschul et al., 1997] search. The PSI-BLAST search was restricted to structures available at the protein data bank (http://www.rcsb.org/pdb/). There were 135 structures gathered in total (Table S1 in Supplementary data) whose homology and membership to the $\alpha$-amylase family (the Glycoside Hydrolase Family 13, GH13) was guaranteed. After the sampling, the methods mentioned above were applied to the dataset and analyzed with $F D$ and $F D_{s}$.

\section{Statistical analysis of protein structural data}

A multidimensional scaling (principal coordinates) analysis and $F D_{s}$ estimation and analysis were performed to two real datasets. The first one, as described above, is a dataset of 135 structures of the $\alpha$-Amylase family. With this dataset, the variation observed is assumed to be driven by the evolutionary history of the structure.

The second dataset is a molecular dynamic simulation of the NiemannPick, type C1 N-terminal domain (NPC1), in solution. The software GROMACS 4.5 [Hess et al., 2008] was used with the force fields OPLS-AA/L [Jorgensen and Tirado-Rives, 1988] for the protein, and the TIP3P [Jorgensen et al., 1983] for the water molecules. The data was collected every 20 picoseconds for 100 nanoseconds discarding the first 10 nanoseconds of simulation to achieve stability. This process was performed using a 24-core GPU-enabled workstation. Each sample was treated as an individual observation for the subsequent analysis, and the data are extracted and processed as explained in sections above. In this dataset two simulations were performed: with Cholesterol (NPC1's ligand) bound to the structure, and another one without the ligand.

\section{Results and Discussion}

A protein fold can be essentially defined as a 3D geometric shape. Sequence analyses help to understand some trends, but explain little about geometry. GM can be used to perform shape analysis from a geometric point of view. It also can be used to give insight into the phylogenetic relationships of the structures rather than the sequences. However, the application of GM to protein structures is not trivial. The scaling component of the Procrustes 
analysis has no conceptual equivalent for proteins. Since organisms grow, it makes sense to extract the size effect on shape in order to compare young with adults. On the other hand, in proteins the atoms do not stretch or grow, and therefore scaling [as proposed in Adams and Naylor, 2000, 2003] is not appropriate.

In [Adams and Naylor, 2000] and [Adams and Naylor, 2003] proposal, they:

- Abstract a residue as a landmark.

- Determine the homologous residues, using ClustalW [Thompson et al., 1994].

- Delete non-homologous sites.

- Perform morphometric analyses.

The use of sequence alignment without structural information to infer structural homology is not accurate since the amount of gaps that can be allowed in a loop region can be different than in other regions of the protein [Kann et al., 2005, Kjer et al., 2007], and therefore the definition of structural homology can be different as well. Moreover, since structures are more conserved than sequences, the alignment based on the structures allows a more reliable asssignment of homology in more distant clades [Wohlers et al., 2012].

In contrast, here we used protein structural alignment which has been worked on extensively [Kolodny et al., 2005, Hasegawa and Holm, 2009, Poleksic, 2011, Joseph et al., 2011, Shibberu et al., 2012]. In particular we used a flexible structure alignment method [MATT; Menke et al., 2008]. This approach strips out rotational and translational information as well as the variability induced by flexible hinges, which has been shown to be more accurate than rigid body superimpositions in the assignment of homology [Menke et al., 2008, Konc and Janežič, 2010, Nguyen et al., 2011, Daniluk and Lesyng, 2011, Joseph et al., 2012, Shah and Sahinidis, 2012, among others].

The abstraction of the residues and landmarks is similar to that in [Adams and Naylor, 2000] and [Adams and Naylor, 2003]; however, those papers did not fully describe the abstraction. 


\section{The effect of scaling in GPS: Insights form HOMSTRAD and SABmark superfamily databases}

In protein structure datasets it is expected that the scaling does not play a major role in the alignment of structures. To test such expectation, a correlation test between the two approaches was performed. The Homstrad dataset showed a correlation coefficient of 0.998, significant $($ pval $<0.001)$, and with an $R^{2}$ of 0.997 . The SABmark dataset also showed a significant and high correlation coeficient $(r=0.994$, pval $<0.001)$ with an $R^{2}$ of 0.987 .

We have shown here not only that scaling is not conceptually acceptable in the context of protein structures (fixed lengths of atomic bonds), but that doing so won't significantly affect the results, supported in high and significant correlation coefficients. From this point further, all GPS analyses made here will be referred as non-scaled GPS.

\section{Comparing MATT flexible alignment and GPS: Results from the HOMSTRAD and SABmark super family databases}

To be able to compare between the MStA and GPS the two types of analyses have to be done independently but comparatively (e.g, using the same variables). However, GPS requires the assignment of homology of the landmarks. As mentioned in Methods, this homology is estimated by the multiple structural alignment. The GPS is an alignment itself, so providing a starting alignment might bias the result of the superimposition. To address this bias, the protein structures are aligned using MATT [Menke et al., 2008] for both approaches and the homologous residues' index for each structure is recorded. For the GPS, the coordinates of the residues corresponding to the recorded indexes are used. This process is performed for each independent structure, therefore avoiding (or at least diminishing) the bias.

Since GPS aligns the structures based only on rotation and translation, it is logical to think of it as a rigid body superimposition. It does not allow any deformation of the structure (shape), like the flexibility that softwares like MATT have. Therefore, it is plausible to hypothesize that a flexible alignment will do better than GPS, as they do against non-flexible alignments [Menke et al., 2008, Konc and Janežič, 2010, Nguyen et al., 2011, Daniluk and Lesyng, 2011, Joseph et al., 2012, Shah and Sahinidis, 2012, among others]. Other flexible structural alignment softwares have shown a slightly better performance than MATT, however, the improvements are not 
significant [Joseph et al., 2012] and MATT returns more core residues than most of its competitors, as well as a statistical test of the "goodness" of the alignment [Menke et al., 2008].

In both databases (Homstrad and SABmark), the correlation coefficients were found to be greater than 0.99 , showing that there is no difference in the use of either approach. This was an unexpected result since if a protein structure is allowed to bend, it is reasonable to expect a better fit. For the datasets explored this seems not to be the case. This observation can be explained if most of the analyzed datasets comprise single domain (which they do) proteins and are therefore more likely to behave as rigid bodies. Also, since GPS depends on the definition of homology inferred by an alignment provided by MATT, GPS could be functioning as a secondary alignment.

Because of this lack of difference, MATT alone is used in the rest of this manuscript.

\section{Form difference: Insights into residue variation}

A sibling field to GM, Dysmorphometrics [Claes et al., 2012], can be used to explore the impact of outlier variables. Dysmorphometrics is in summary, "the modeling of morphological abnormalities" [Claes et al., 2012]. Such exploration can be performed by means of corrected maximum likelihood estimates approach [as in Claes et al., 2012] or by means of the Euclidean distance matrix analysis approach [Claude, 2008]. Here we use the latter since it is simpler and requires fewer parameters to be set.

Claude [2008], after the work of Lele and Richtsmeier [1992], proposed a way to examine the influence of landmarks in shape difference by calculating the sum of residuals from the median for each landmark given the FDM matrix. The landmarks that influence the most difference in shape would have a higher score which can be mapped to the given shape. In the context of geometric morphometrics, the FDM accounts for landmarks that have an excessive variation among a pair of shapes.

\section{Shape simulation}

To illustrate this effect, a hexagon simulation was performed where the variation among the observations is controlled. Figure 1 shows the plotting of 500 samples in this simulation. 


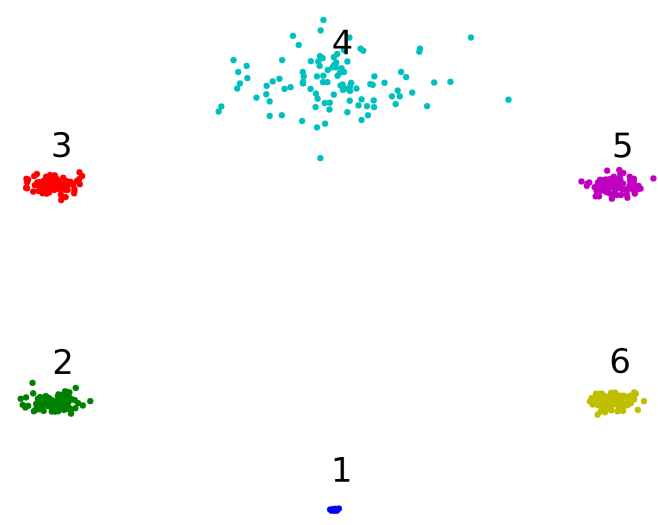

Figure 1: Hexagon simulation. Points 2, 3, 5, and 6 have a standard deviation of 0.05 , while point 1 (low) have 0.005 and point 4 (high) have an standard deviation of 0.2 . This plot was performed using the python library Matplotlib [Hunter, 2007].

Here a point with high variation (point 4 in Figure 1) and a point with minimal variation (point 1 in Figure 1) are introduced, along with four other points exhibiting an average variation in the context of geometric morphometrics [von Cramon-Taubadel et al., 2007].

Table 1: Scaled FD values for the simulation illustrated in Figure 1.

\begin{tabular}{ccc}
\hline Landmark & Standard deviation & Scaled FD \\
\hline 1 & 0.005 & -1 \\
2 & 0.05 & -0.78 \\
3 & 0.05 & -0.33 \\
4 & 0.2 & 1 \\
5 & 0.05 & -0.28 \\
6 & 0.05 & -0.83 \\
\hline
\end{tabular}


Table 1 and Figure 1 show that the $F D_{s}$ represents the overall influence of a point in the shape. Negative values of $F D_{s}$ mean a more conserved point, being -1 the most conserved. On the other hand, positive $F D_{s}$ values are related to more influential points, being +1 the most variable. From Table 1, one can also see that the "average" points are closer to the least variation than to the highest one, therefore displaying a negative tendency. At fist sight it might seem trivial to use the $F D_{s}$ since the standard deviation $(s d)$ seems to correlate with it. However, $F D_{s}$ represents the influence of a landmark relative to the overall shape, as opposed to the $s d$ which represents the variation at a single variable level. Variables with high $s d$ s in a medium-sd neighbourhood will have very high $F D_{s}$, while very low-sd points will have low $F D_{s}$. This suggests that the $s d$ can be used as proxy for $F D$. However, in a setting where all the points have high $s d$, the relation between $s d$ and $F D$ is not as direct. Moreover, for the relationship between $s d$ and $F D_{s}$ to be proportional, a model of isotropic variation in all dimensions is needed. Such model assumes an equal amount of variation at each landmark and at each dimension in each landmark. It also assumes that landmarks are independent, which is not a fair assumption in most shape analysis [Klingenberg, 2003].

Non-scaled FD can be also used to screen a set of points in a shape to look for "Pinocchio" outliers, using statistical tests as the Dixon's Q test or the Grubbs' test for outliers.

\section{$\alpha$-Amylase simulation and evolutionary dataset}

To test the form difference (FD) in more complex shapes, the $\alpha$-Amylase dataset was used. First a simulation using the porcine pancreatic amylase (1PPI) was used to control the variation in two random residues (see section Analysis of the deformation in superimposed structures/shapes for details). Figure 2A shows the result of the FD analysis on the simulation.

Here we can see which residues contribute the most and the least to the overall shape, as well as their relative position.

As expected for the real dataset of homologs (Figure 2B), the most variable (and therefore with positive $F D_{s}$ values) residues are in located in loops. The residue Arg10 was the most variable, and is also located at the beginning of the chain after the signal peptide; therefore its high variability may be explained by this location in the primary structure. The residue with the least variation is Phe136 which is also found in a loop. The Phe136 residue is 


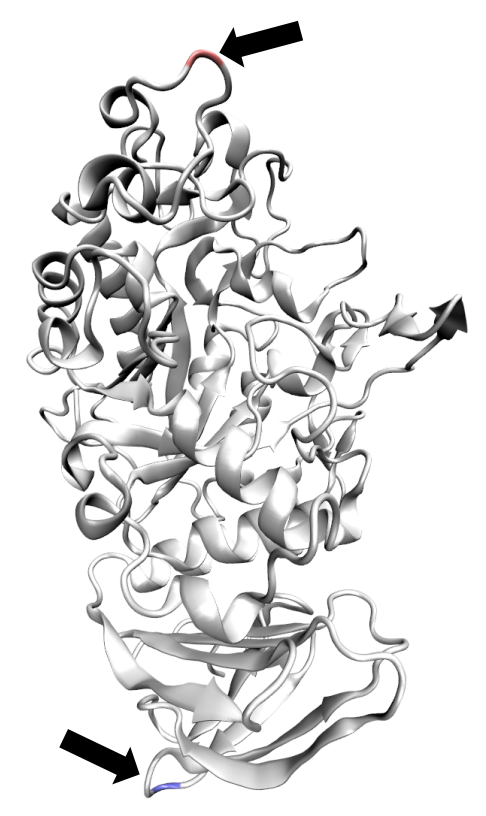

(a) Simulation

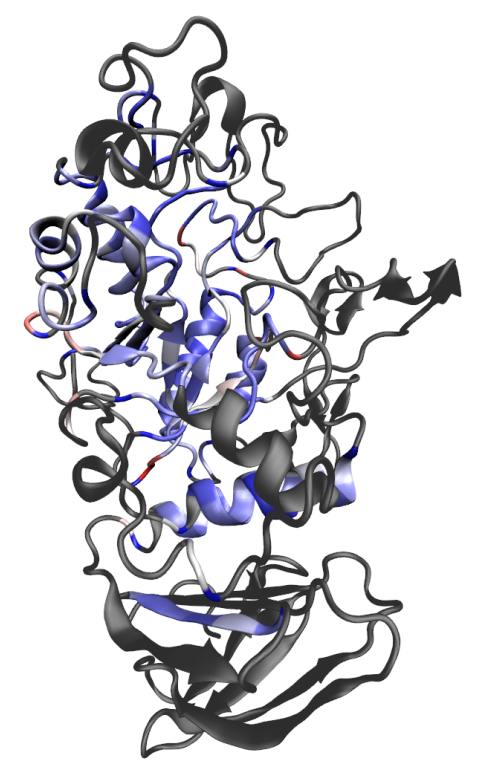

(b) 135 homologs

Figure 2: $F D_{s}$ values mapped in the porcine pancreatic Amylase structure (PDB code: 1PPI). Red represents the highly variable, while blue the least variable. A) A simulation of the values (The locations of the points are selected at random and do not represent any biological meaning). The highest and lowest $F D_{s}$ are represented in red and blue respectively and pointed by arrows. Here the color scale was offset by 0.5 and the midpoint was set at 0.01 for visualization. B) The $F D_{s}$ for a dataset of 135 structures, gathered with a PSI blast seeded with a PFAM seed alignment. The structure used for FD mapping is the porcine pancreatic amylase (PDB code: 1PPI). The grey chain correspond to the non homologous section of the 1PPI with respect to the alignment. Both figures were rendered with VMD v1.91 [Humphrey et al., 1996].

not reported to bind to ligands or be involved in catalytic activity. However, this residue is within $15 \AA$ (in a $C_{\alpha}-C_{\alpha}$ perspective) of metal and ligand binding residues and it is also highly conserved. 


\section{NPC1 dataset: Analysis of the residue variation in the context of ligand binding}

The Niemann-Pick, Type C-1 protein (NPC1) binds cholesterol and oxysterols [Infante et al., 2008] and has an important role in the metabolism of cholesterol and other lipids. Defects in NPC1 cause malfunction of the cholesterol, sphingolipids, phospholipids, and glycolipids pathways. The protein contains 1278 residues, with 13 membrane helices and three large loops that project to the lumen of lysosomes [Infante et al., 2008]. The first luminal domain is the N-terminal domain, which comprises approximately 240 amino acids. This is a lumen domain, and therefore not in the trans-membrane region of the protein.

To check the overall contribution of each of the residues to the deformations during the simulation, equation 6 was applied and results were mapped on the original protein structure. Figure 3 shows that once the cholesterol is bound to the NPC1 (Figure 3A), most of the higher FD residues are not contributing to the deformation. It seems that most residues' movement is been held in place by interactions with the ligand.
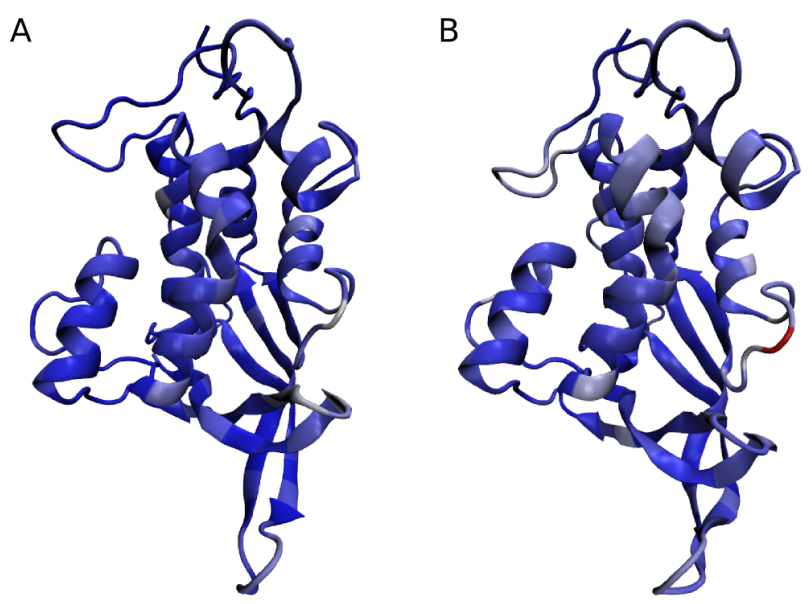

Figure 3: FDM analysis of the NPC1 N-terminal domain (pdbcode 3GKH) with $3 \mathrm{~A}$ and without $3 \mathrm{~B}$ ligand. The color represents the $F D$, red being a higher score and blue the lowest. For comparison, the scale was set from the minimum FD value in both, to the maximum between the two. In this particular case, lower $F D$ values (therefore the least variables) dominate the scale and the most influential is shown in red. 
The opposite behaviour can be seen in Figure 3B. When cholesterol is not bound to it, the residues in charge of the cholesterol intake/outtake are more movable. Therefore, these residues are responsible for most if the deformation. This makes sense if the binding pocket must be flexible enough to open and close upon binding with the ligand.

\section{GM-like analysis of protein data: An example from the multidimensional scaling}

$\alpha$-Amylase homologs: Geometry, function and structural similarity

After aligning the structures and applying the methods exposed in section Material and methods, a principal coordinate analysis (PCoA) was performed to the resulting landmark data (Figure 4). Analyzing the geometry of the protein structures using a PCoA can give us insight into the relationships of such shapes. This procedure test for differences in the structures being compared, and will show patterns of clustering based on their geometric similarity which in turn might be highly correlated with the functional similarity [Wright and Dyson, 1999].

The PCoA of the multiple structure alignment (Figure 4), showed seven distinct and tightly clustered groups:

\section{Chloride-dependent $\alpha$-Amylases}

The first group corresponds to the Cloride-dependent $\alpha$-Amylases (with amylase function or EC \# 3.2.1.1). The similarity among these $\alpha$ amylases is not a new observation. D'Amico et al. [2000] identified potential chloride-dependent amylases, based on the chloride allosteric activation positives: A) PPA or porcine (Sus scrofa) pancreatic $\alpha$ amylase; B) HPA or human (Homo sapiens) pancreatic $\alpha$-amylase; C) TMA or Tenebrio molitor (mealworm) $\alpha$-amylase; and D) AHA or Pseudoalteromonas haloplanktis (before classified as Alteromonas) $\alpha$ amylase. They showed that the side chains of residues Arg195, Asn298 and Arg/Lys337 (PPA numbering), are related to chloride ion binding capabilities [Da Lage et al., 2004].

\section{Thermal/alkalostable or calcium independent $\alpha$-Amylases}

The next tightly defined group in Figure 4 are structures that show higher stability in extreme $\mathrm{PH}$ and/or thermal conditions or are calcium independent. As shown in Figure 4 there is a subgroup of mutants 
with higher structural shift from the main group. In this sub-cluster three thermo-stable $\alpha$-amylases (EC \# 3.2.1.1) mutants from the genus Bacillus can be found. In two of the three cases (3DC0, Rahimzadeh et al., 2012; 1BF2, Fujimoto et al., 1998) the directed mutagenesis was performed to increase thermal stability. In the case of $1 \mathrm{UA} 7$, it is a mutant of the catalytic site that is not supposed to change stability or function with respect to the wild type [Kagawa et al., 2003]. However, this structure was modeled using $1 \mathrm{BF} 2$, and the clustering observed in its structure suggests a higher performance or thermal-stability than other non-chloride binding bacterial amylases. The rest of the group includes $\alpha$-amylases that exhibit higher thermal/alkaline stability or enhanced efficiency with respect to other amylases of similar function ( $\alpha$-1,4-glucan-4-glucanohydrolase, EC \# 3.2.1.1) [Shimi et al., 2008]. Most of these structures were created by directed mutagenesis to enhance their industrial applicability by either increasing their thermal or alkaline resistance [Hwang et al., 1997, Machius et al., 1998, Brzozowski et al., 2000, Machius et al., 2003, Lyhne-Iversen et al., 2006, Shirai et al., 2007, Shimi et al., 2008, Alikhajeh et al., 2010] or to make them calcium independent [Prakash and Jaiswal, 2010]. There is also a structure with a different enzymatic classification, the maltohexaosidase from Bacillus licheniformis (1WP6; glucan 1,4- $\alpha$-maltohexaosidase or EC \# 3.2.1.98). Despite carrying a slightly different reaction, its native state exhibit higher alkaline stability than other native amylases [Kanai et al., 2004a].

\section{Cyclomaltodextrinase-like $\alpha$-Amylases}

The Cyclomaltodextrinase + Neopullulanase + Maltogenic Amylases group (Figure 4) includes enzymes classified in seemingly different functional groups (Cyclomaltodextrinases EC \# 3.2.1.54; maltogenic amylases, EC \# 3.2.1.133; neopullulanases EC \# 3.2.1.135) that can hydrolyze cyclomaltodextrins efficiently [Park et al., 2000] but not starch and pullulan as efficiently [Lee et al., 2002]. However, Lee et al. [2002] have shown that despite their different enzyme codes, there are no thoroughly documented differences in the literature about their function or structure. They proposed to unify this group under the same enzyme number and the same name (Cyclomaltodextrinases). The result, shown in Figure 4, suggests that this is the case given our clustering based on shape. It is important to mention that this Cyclomal- 
todextrinase group has to be distinguished from the Cyclomatodextrin glucotransferase group, since those are extracellular enzymes whereas the Cyclomaltodextrinase-like $\alpha$-Amylases are intracellular[Lee et al., 2002].

Cyclomaltodextrinase-like $\alpha$-Amylases with structural shifts The Neopullunanases with structurally shifting mutations groups is a subset of the Cyclomaltodextrinases described above. They carry the same functions (mainly Neopullunanse; EC \# 3.2.1.135), but have been subjected to mutagenesis either for binding studies (i.e. $2 \mathrm{FH} 8$ and 2FHB ; Mikami et al., 2006) or to inactivate the enzyme using sitedirected mutagenesis [Ohtaki et al., 2001, Yokota et al., 2001, Ohtaki et al., 2004, Mizuno et al., 2005]. As can be seen in Figure 4, even a small number of substitution cause structural shifts that can be identified by means of a PCoA.

\section{Cyclomatodextrin glucotransferases-like $\alpha$-Amylases}

This group is composed entirely of bacterial (mainly from the genus Bacillus) $\alpha$-Amylases that catalyze the conversion of starch to cyclodextrins (EC \# 2.4.1.19) [Kanai et al., 2004b]. As can be seen in Figure 4, it is a tightly defined group markedly different from the rest. These differences can be explained by the presence of four aromatic residues that are not present in other amylases and are strongly associated with the protein function [Tonkova, 1998, Kanai et al., 2004b].

\section{Maltotetraose-forming exo-amylase}

This is singleton group, containing the structure 1GCY [Mezaki et al., 2001] from Pseudomonas stutzeri. It is a glucan 1,4-alpha-maltotetraohydrolase (EC \# 3.2.1.60) that works hydrolyzing amylaceous polysaccharides and removing successive maltotetraose residues from the non-reducing chain ends [Fleischmann et al., 2004]. It behaves as an exo-amylase and structural differences with respect to endo-amylases were expected, to be able to remove the residues at the end of the chain instead of just breaking the 1-4 glycosidic linkages. The PCoA in Figure 4 expresses this differences by showing a distance between this structure with the "endo-amylases".

\section{Sucrose-related isomerases and hydrolases}

This group contains the structures mainly classified as Sucrose glucosyl- 
mutases (or Isomaltulose synthase EC \# 5.4.99.11)[Fleischmann et al., 2004]. However, it also contains three structures (namely 2ZIC, 2ZID, and 4AIE), with Glucan 1,6-alpha-glucosidase (EC \# 3.2.1.70) function [Kim et al., 2005, Hondoh et al., 2008, Møller et al., 2012], from which the 2ZIC and 2ZID are have been subjected to directed mutagenesis to improve their catalytic efficiency. This group also harbors an $\alpha$-glucosidase (2ZE0; Shirai et al., 2008) and an Oligo-1,6-glucosidase (Isomaltase; 1AXH) mutant [Yamamoto et al., 2011]. The rest of structures there are Isomaltulose synthases (EC \# 5.4.99.11), including three (4GIN,4GI6, and 4H2C) misannotations (inexistent EC \# 5.4.11.99 instead of EC \# 5.4.99.11). Despite the somewhat disparity in function, they all are classified in the GH13 family [Cantarel et al., 2009, Svensson and Janecek, 2013], and the results shown in Figure 4 suggest a high structural similarity. 


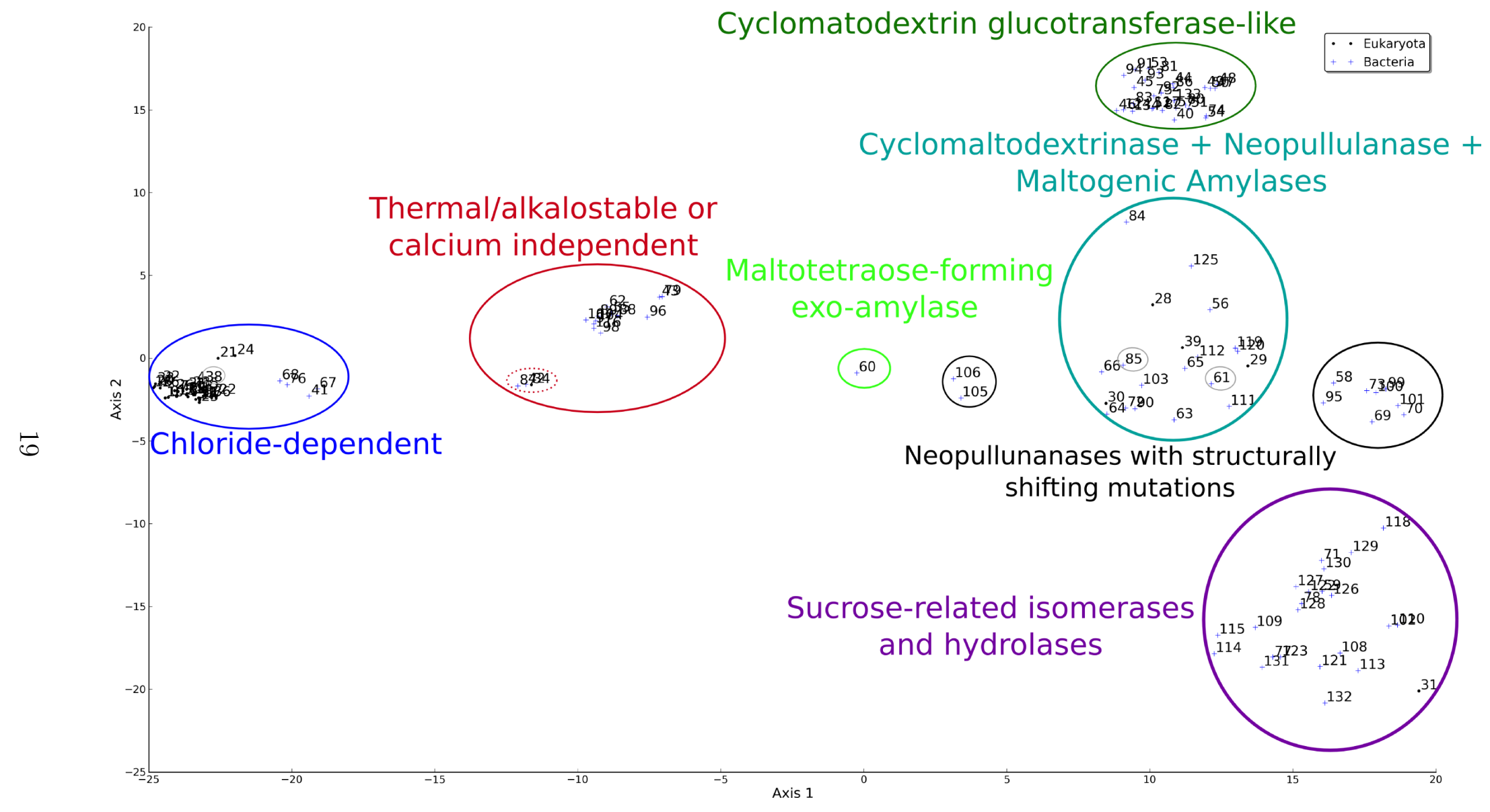

Figure 4: Principal Co-ordinates Analysis (PCoA) of 135 protein crystals of the $\alpha$-Amylase. The circled groups show clusters of structural similarity. The PCoA was performed in R [R, 2011] and modified with a python script using Matplotlib [Hunter, 2007] library. 
As can be seen, the principal coordinate analysis of protein structures is tightly correlated with function, and might give some insights into missannotations or potential functional discoveries. This can be useful for the classification of proteins. This clustering scheme might show an apparent correlation with phylogeny. However, this approach showed to be sensitive to structural changes. It identified even mutants from the wild type if a structural shift has occurred. This may suggest that this approach is capturing more structural similarities than only phylogenetic ones. It would be interesting to explore phylogenetic signal free variables to test such a hypothesis, but even now this approach seems to be robust to find functional/structural groups.

\section{NPC1 molecular dynamic data}

The NPC1 N-terminal domain dataset was explored using a PCoA (Figures $5 \mathrm{~A}$ and $5 \mathrm{~B}$ ) to analyze the trajectories as a composite measure of overall structures (principal coordinates).
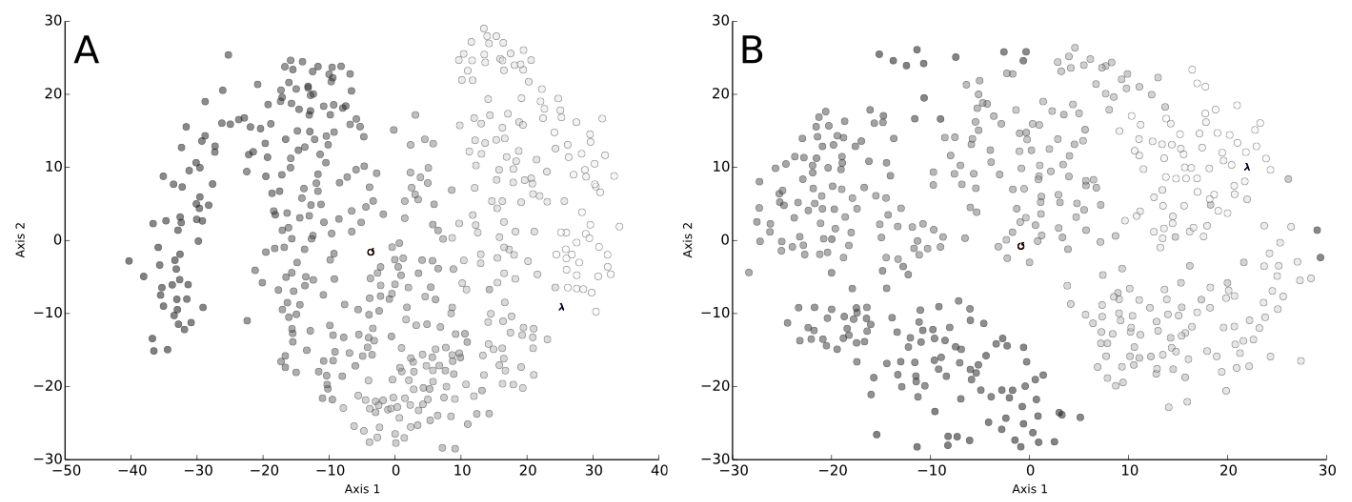

Figure 5: Principal coordinate analysis of 495 snapshots from 100 ns of molecular dynamic simulations of the NPC1 N-terminal domain without (5A) and with $(5 \mathrm{~B})$ cholesterol bound to it. The gray scale is proportional to the time of the simulation getting progressively lighter as the simulation develops. The symbols $\circlearrowleft$ and $\lambda$ represent the starting and final points respectively

This exploratory analysis seems to indicate that a burn-in of 10 nanoseconds and a sampling every 20 ps achieves stability in both cases (Figure 5). The overall structure seems to sample the space around the initial point 
(Figure 5). However, the simulation with the cholesterol (5B) seems to have a narrower shift (as per first axis) than the ligand-free (5A). The shifts here are represented for a wider move in the principal coordinates space. This technique can be used to see if clusters of structures appear, and therefore a deviation from equilibria or representation of multiple states in a molecular dynamic simulation. This method in conjunction with the energy profile and a clustering scheme, can provide useful information about the dynamic properties of proteins.

\section{Conclusions}

Here we have shown how to apply GM-like methods to the protein structure variation analysis problem. We have also shown that by using GM-like methods, it is possible to explore patterns in both evolutionary and dynamic samplings. Due to the scarcity of space, a full application of different statistical techniques could not be done. However, the application of techniques, such as linear discriminant analysis, support vector machine, random forest, multivariate analysis of variance, different types of regression, among others, are simple extensions of what we have shown here. Such techniques might help in classification of proteins (structurally or phylogenetically) as well as characterizing the variation associated with different parameters in the structural space.

\section{Acknowledgements}

The authors thank professors Andrew Roger, Jan K. Rainey, and Edward Susko for useful insights. Also, the members of the Blouin lab for the critical review of the manuscript and to Liz Mackay for the language editing the manuscript. This study was funded by NSERC through the grant No. 120504858. This work was partially supported by The Departamento Administrativo de Ciencia y Tecnología - Colciencias (Colombia) through the CALDAS scholarship. 
Table S1: PDB codes of the $\alpha$-amylase homologues, the species from it was crystallized, and its corresponding equivalence number in the PCoA plot in Figure 4. Only the chain A, corresponding to the catalytic domain, was used.

\section{Supplementary data}

\section{References}

D. C. Adams and G. J. P. Naylor. A new method for evaluating the structural similarity of proteins using geometric morphometrics. In S. Miyano, R. Shamir, and T. Takagi, editors, Currents in Computational Molecular Biology, volume 30 of Frontiers Science Series, pages 120-121, Tokyo, 2000. Universal Academy Press.

Dean C. Adams and Gavin J. P. Naylor. A comparison of methods for assessing the structural similarity of proteins. In Mathematical Methods for Protein Structure Analysis and Design, pages 109-115, 2003. doi: 10. 1007/978-3-540-44827-3_6.

Dean C. Adams, F. James Rohlf, and Dennis E. Slice. Geometric morphometrics: Ten years of progress following the 'revolution'. Italian Journal of Zoology, 71(1):5-16, 2004. doi: 10.1080/11250000409356545. URL http://www . tandfonline.com/doi/abs/10.1080/11250000409356545.

Jahan Alikhajeh, Khosro Khajeh, Bijan Ranjbar, Hossein Naderi-Manesh, Y. H. Lin, Enhung Liu, H. H. Guan, Y. C. Hsieh, Phimonphan Chuankhayan, Y. C. Huang, et al. Structure of bacillus amyloliquefaciensamylase at high resolution: implications for thermal stability. Acta Crystallographica Section F: Structural Biology and Crystallization Communications, 66(2):121-129, 2010.

S. F. Altschul, T. L. Madden, A. A. Schäffer, J. Zhang, Z. Zhang, W. Miller, and D. J. Lipman. Gapped blast and psi-blast: a new generation of protein database search programs. Nucleic acids research, 25(17):3389-3402, 1997. doi: 10.1093/nar/25.17.3389.

Andrzej M. Brzozowski, David M. Lawson, Johan P. Turkenburg, Henrik Bisgaard-Frantzen, Allan Svendsen, Torben V. Borchert, Zbigniew Dauter, 
Keith S. Wilson, and Gideon J. Davies. Structural analysis of a chimeric bacterial $\alpha$-amylase. high-resolution analysis of native and ligand complexes. Biochemistry, 39(31):9099-9107, 2000. doi: 10.1021/bi0000317.

Brandi L. Cantarel, Pedro M. Coutinho, Corinne Rancurel, Thomas Bernard, Vincent Lombard, and Bernard Henrissat. The carbohydrate-active enzymes database (cazy): an expert resource for glycogenomics. Nucleic acids research, 37(suppl 1):D233-D238, 2009. doi: 10.1093/nar/gkn663.

Peter Claes, Katleen Daniels, Mark Walters, John Clement, Dirk Vandermeulen, and Paul Suetens. Dysmorphometrics: the modelling of morphological abnormalities. Theor Biol Med Model, 9:5, 2012. doi: 10.1186/1742-4682-9-5.

Julien Claude. Morphometrics with $R$. Use R! Springer, 2008. ISBN 9780387777894 . URL http://www.worldcat.org/isbn/9780387777894.

J. L. Da Lage, Georges Feller, and Š Janeček. Horizontal gene transfer from eukarya to bacteria and domain shuffling: the $\alpha$-amylase model. Cellular and Molecular Life Sciences CMLS, 61(1):97-109, 2004. doi: 10.1007/ s00018-003-3334-y.

S. D'Amico, C. Gerday, and G. Feller. Structural similarities and evolutionary relationships in chloride-dependent $\alpha$-amylases. Gene, 253(1):95-105, 2000. doi: 10.1016/S0378-1119(00)00229-8.

Paweł Daniluk and Bogdan Lesyng. A novel method to compare protein structures using local descriptors. BMC Bioinformatics, 12(1):344, 2011. doi: 10.1186/1471-2105-12-344.

I. L. Dryden. Shapes package. $R$ Foundation for Statistical Computing, Vienna Contributed package, 2011.

I. L. Dryden and Kanti V. Mardia. Statistical Shape Analysis. Wiley, Chichester, 1 edition, 1998. ISBN 9780471958161.

R. D. Finn, J. Mistry, J. Tate, P. Coggill, A. Heger, J. E. Pollington, O. L. Gavin, P. Gunasekaran, G. Ceric, K. Forslund, Liisa Holm, Erik L. L. Sonnhammer, Sean R. Eddy, and Alex Bateman. The pfam protein families database. Nucleic acids research, 38(suppl 1):D211 - D222, 2010. doi: $10.1093 /$ nar/gkp985. 
Astrid Fleischmann, Michael Darsow, Kirill Degtyarenko, Wolfgang Fleischmann, Sinéad Boyce, Kristian B. Axelsen, Amos Bairoch, Dietmar Schomburg, Keith F. Tipton, and Rolf Apweiler. Intenz, the integrated relational enzyme database. Nucleic acids research, 32(suppl 1):-434, 2004. doi: $10.1093 /$ nar/gkh119.

Zui Fujimoto, Kenji Takase, Nobuko Doui, Mitsuru Momma, Takashi Matsumoto, and Hiroshi Mizuno. Crystal structure of a catalytic-site mutant $\alpha$-amylase from bacillus subtilis complexed with maltopentaose. Journal of Molecular Biology, 277(2):393-407, 1998. ISSN 0022-2836. doi: 10.1006/jmbi.1997.1599.

Hitomi Hasegawa and Liisa Holm. Advances and pitfalls of protein structural alignment. Current Opinion in Structural Biology, 19(3):341-348, 2009. ISSN 0959-440X. doi: 10.1016/j.sbi.2009.04.003. URL http://www . sciencedirect.com/science/article/pii/S0959440X09000621.

B. Hess, C. Kutzner, D. van der Spoel, and E. Lindahl. Gromacs 4: Algorithms for highly efficient, load-balanced, and scalable molecular simulation. Journal of chemical theory and computation, 4(3):435-447, 2008. doi: $10.1021 /$ ct700301q.

Hironori Hondoh, Wataru Saburi, Haruhide Mori, Masayuki Okuyama, Toshitaka Nakada, Yoshiki Matsuura, and Atsuo Kimura. Substrate recognition mechanism of $\alpha$-1, 6-glucosidic linkage hydrolyzing enzyme, dextran glucosidase from Streptococcus mutans. Journal of molecular biology, 378 (4):913-922, 2008. doi: 10.1016/j.jmb.2008.03.016.

William Humphrey, Andrew Dalke, and Klaus Schulten. Vmd - visual molecular dynamics. Journal of Molecular Graphics, 14:33-38, 1996. doi: 10.1016/0263-7855(96)00018-5.

J. D. Hunter. Matplotlib: A 2d graphics environment. Computing In Science \& Engineering, 9(3):90-95, 2007. doi: 10.1109/MCSE.2007.55.

Kwang Yeon Hwang, Hyun Kyu Song, Changsoo Chang, Jungkyu Lee, S. Y. Lee, K. K. Kim, Senyon Choe, Robert M. Sweet, and S. W. Suh. Crystal structure of thermostable alpha-amylase from bacillus licheniformis refined at 1.7 a resolution. Molecules and cells, 7(2):251-258, 1997. 
Rodney E Infante, Arun Radhakrishnan, Lina Abi-Mosleh, Lisa N Kinch, Michael L Wang, Nick V Grishin, Joseph L Goldstein, and Michael S Brown. Purified npc1 protein: Ii. localization of sterol binding to a 240amino acid soluble luminal loop. J. Biol. Chem., 283(2):1064-75, 2008. doi: $10.1074 /$ jbc.M707944200.

Eric Jones, Travis Oliphant, and Pearu Peterson. Scipy: Open source scientific tools for python. http://www. scipy. org/, 2001.

William L. Jorgensen and Julian Tirado-Rives. The opls [optimized potentials for liquid simulations] potential functions for proteins, energy minimizations for crystals of cyclic peptides and crambin. Journal of the American Chemical Society, 110(6):1657-1666, 1988. doi: 10.1021/ja00214a001.

William L. Jorgensen, Jayaraman Chandrasekhar, Jeffry D. Madura, Roger W. Impey, and Michael L. Klein. Comparison of simple potential functions for simulating liquid water. The Journal of chemical physics, 79 (2):926-935, 1983.

Agnel Praveen Joseph, N. Srinivasan, and Alexandre G. de Brevern. Improvement of protein structure comparison using a structural alphabet. Biochimie, 93(9):1434-1445, 2011. ISSN 0300-9084. doi: 10.1016/j.biochi. 2011.04.010. URL http://www.sciencedirect.com/science/article/ pii/S0300908411001295.

Agnel Praveen Joseph, Narayanaswamy Srinivasan, and Alexandre G. de Brevern. Progressive structure-based alignment of homologous proteins: Adopting sequence comparison strategies. Biochimie, 94(9):2025-2034, 2012. ISSN 0300-9084. doi: 10.1016/j.biochi.2012.05.028. URL http:// WWw.sciencedirect.com/science/article/pii/S0300908412002167.

Masayuki Kagawa, Zui Fujimoto, Mitsuru Momma, Kenji Takase, and Hiroshi Mizuno. Crystal structure of Bacillus subtilis $\alpha$-amylase in complex with acarbose. Journal of Bacteriology, 185(23):6981-6984, 2003. doi: 10.1128/JB.185.23.6981-6984.2003.

Ryuta Kanai, Keiko Haga, Toshihiko Akiba, Kunio Yamane, and Kazuaki Harata. Biochemical and crystallographic analyses of maltohexaoseproducing amylase from alkalophilic bacillus sp. 707. Biochemistry, 43 (44):14047-14056, 2004a. 
Ryuta Kanai, Keiko Haga, Toshihiko Akiba, Kunio Yamane, and Kazuaki Harata. Role of phe283 in enzymatic reaction of cyclodextrin glycosyltransferase from alkalophilic bacillus sp. 1011: Substrate binding and arrangement of the catalytic site. Protein science, 13(2):457-465, 2004b. doi: $10.1110 /$ ps.03408504.

Maricel G Kann, Paul A Thiessen, Anna R Panchenko, Alejandro A Schäffer, Stephen F Altschul, and Stephen H Bryant. A structure-based method for protein sequence alignment. Bioinformatics, 21(8):1451-6, 2005. doi: 10.1093/bioinformatics/bti233. URL http://bioinformatics . oxfordjournals.org/content/21/8/1451.abstract.

H. K. Kim, J. W. Liu, Paul D. Carr, and David L. Ollis. Following directed evolution with crystallography: structural changes observed in changing the substrate specificity of dienelactone hydrolase. Acta Crystallographica Section D: Biological Crystallography, 61(7):920-931, 2005.

Karl M Kjer, Joseph J Gillespie, and Karen A Ober. Opinions on multiple sequence alignment, and an empirical comparison of repeatability and accuracy between poy and structural alignment. Syst. Biol., 56(1): 133-46, 2007. doi: 10.1080/10635150601156305. URL http://sysbio. oxfordjournals.org/content/56/1/133. short.

C.P. Klingenberg. Quantitative genetics of geometric shape: heritability and the pitfalls of the univariate approach. Evolution, 57(1):191-195, 2003.

Rachel Kolodny, Patrice Koehl, and Michael Levitt. Comprehensive evaluation of protein structure alignment methods: scoring by geometric measures. J. Mol. Biol., 346(4):1173-88, 2005. doi: 10.1016/j.jmb.2004.12.032.

J. Konc and D Janežič. Probis algorithm for detection of structurally similar protein binding sites by local structural alignment. Bioinformatics, 26(9): 1160-1168, 2010. doi: 10.1093/bioinformatics/btq100.

Hee-Seob Lee, Min-Sung Kim, Hyun-Soo Cho, Jung-In Kim, Tae-Jip Kim, JiHye Choi, Cheonseok Park, Heung-Soo Lee, Byung-Ha Oh, and Kwan-Hwa Park. Cyclomaltodextrinase, neopullulanase, and maltogenic amylase are nearly indistinguishable from each other. Journal of Biological Chemistry, 277(24):21891-21897, 2002. doi: 10.1074/jbc.M201623200. 
Subhash Lele and Joan T. Richtsmeier. On comparing biological shapes: Detection of influential landmarks. American Journal of Physical Anthropology, 87(1):49-65, 1992. ISSN 1096-8644. doi: 10.1002/ajpa.1330870106. URL http://dx.doi.org/10.1002/ajpa.1330870106.

Louise Lyhne-Iversen, Timothy J. Hobley, Svend G. Kaasgaard, and Pernille Harris. Structure of bacillus halmapalus-amylase crystallized with and without the substrate analogue acarbose and maltose. Acta Crystallographica Section F: Structural Biology and Crystallization Communications, 62 (9):849-854, 2006.

Mischa Machius, Nathalie Declerck, Robert Huber, and Georg Wiegand. Activation of bacillus licheniformis $\alpha$-amylase through a disorder $\rightarrow$ order transition of the substrate-binding site mediated by a calcium-sodiumcalcium metal triad. Structure, 6(3):281-292, 1998. ISSN 0969-2126. doi: 10.1016/S0969-2126(98)00032-X.

Mischa Machius, Nathalie Declerck, Robert Huber, and Georg Wiegand. Kinetic stabilization of bacillus licheniformis $\alpha$-amylase through introduction of hydrophobic residues at the surface. Journal of Biological Chemistry, 278(13):11546-11553, 2003. doi: 10.1074/jbc.M212618200.

Matthew Menke, Bonnie Berger, and Lenore Cowen. Matt: local flexibility aids protein multiple structure alignment. PLoS Comput. Biol., 4(1):e10, 2008. doi: 10.1371/journal.pcbi.0040010.

Yoshihiro Mezaki, Yoshio Katsuya, Michio Kubota, and Yoshiki Matsuura. Crystallization and structural analysis of intact maltotetraose-forming exoamylase from pseudomonas stutzeri. Bioscience, biotechnology, and biochemistry, 65(1):222-225, 2001. doi: 10.1271/bbb.65.222.

Bunzo Mikami, Hiroyuki Iwamoto, Dominggus Malle, Hye-Jin Yoon, Elif Demirkan-Sarikaya, Yoshihiro Mezaki, and Yoshio Katsuya. Crystal structure of pullulanase: evidence for parallel binding of oligosaccharides in the active site. Journal of molecular biology, 359(3):690-707, 2006. doi: 10.1016/j.jmb.2006.03.058.

Masahiro Mizuno, K.azuhiro Ichikawa, Takashi Tonozuka, Akashi Ohtaki, Yoichiro Shimura, Shigehiro Kamitori, Atsushi. Nishikawa, and Yoshiyuki 
Sakano. Mutagenesis and structural analysis of thermoactinomyces vulgarius r-47 $\alpha$-amylase ii (tvaii). Journal of Applied Glycoscience, 52(3): 225-231, 2005. doi: 10.5458/jag.52.225.

Marie S. Møller, Folmer Fredslund, Avishek Majumder, Hiroyuki Nakai, Jens-Christian N. Poulsen, Leila Lo Leggio, Birte Svensson, and Maher Abou Hachem. Enzymology and structure of the gh13_31 glucan 1, 6- $\alpha$-glucosidase that confers isomaltooligosaccharide utilization in the probiotic lactobacillus acidophilus ncfm. Journal of bacteriology, 194(16): 4249-4259, 2012. doi: 10.1128/JB.00622-12.

M. N. Nguyen, K. P. Tan, and M. S. Madhusudhan. Click-topologyindependent comparison of biomolecular 3d structures. Nucleic acids research, 39(suppl 2):-24, 2011. doi: 10.1093/nar/gkr393.

Akashi Ohtaki, Shin Kondo, Yoichiro Shimura, Takashi Tonozuka, Yoshiyuki Sakano, and Shigehiro Kamitori. Role of phe286 in the recognition mechanism of cyclomaltooligosaccharides (cyclodextrins) by Thermoactinomyces vulgaris $\mathrm{r}-47 \alpha$-amylase 2 (tvaii). x-ray structures of the mutant tvaiis, f286a and f286y, and kinetic analyses of the phe286-replaced mutant tvaiis. Carbohydrate Research, 334(4):309-313, 2001. doi: 10.1016/ S0008-6215(01)00190-2.

Akashi Ohtaki, Masahiro Mizuno, Takashi Tonozuka, Yoshiyuki Sakano, and Shigehiro Kamitori. Complex structures of thermoactinomyces vulgaris r47 a-amylase 2 with acarbose and cyclodextrins demonstrate the multiple substrate recognition mechanism. Journal of Biological Chemistry, 279 (30):31033-31040, 2004. doi: 10.1074/jbc.M404311200.

Kwan-Hwa Park, Tae-Jip Kim, Tae-Kyou Cheong, Jung-Wan Kim, Byung$\mathrm{Ha} \mathrm{Oh}$, and Birte Svensson. Structure, specificity and function of cyclomaltodextrinase, a multispecific enzyme of the $\alpha$-amylase family. Biochimica et Biophysica Acta (BBA)-Protein Structure and Molecular Enzymology, 1478(2):165-185, 2000. doi: 10.1016/S0167-4838(00)00041-8.

Aleksandar Poleksic. Optimizing a widely used protein structure alignment measure in expected polynomial time. IEEE/ACM Trans Comput Biol Bioinform, 8(6):1716-20, nov.-dec. 2011. ISSN 1545-5963. doi: 10.1109/ TCBB.2011.122. 
Om Prakash and Nivedita Jaiswal. $\alpha$-amylase: an ideal representative of thermostable enzymes. Applied biochemistry and biotechnology, 160(8): 2401-2414, 2010. doi: 10.1007/s12010-009-8735-4.

Development Core Team R. R: A Language and Environment for Statistical Computing. Vienna, Austria, 2011. URL http://www.R-project.org/. ISBN 3-900051-07-0.

Mahsa Rahimzadeh, Khosro Khajeh, Manoochehr Mirshahi, Mahmood Khayatian, and Robert Schwarzenbacher. Probing the role of asparagine mutation in thermostability of Bacillus kr-8104 $\alpha$-amylase. International Journal of Biological Macromolecules, 50(4):1175-1182, 2012. doi: 10.1016/j.ijbiomac.2011.11.014.

F. J. Rohlf. Morphology, Shape and Phylogeny, chapter Geometric Morphometrics and Phylogeny, pages 175-193. Taylor and Francis, New York, 2002 .

F. James Rohlf and Dennis Slice. Extensions of the procrustes method for the optimal superimposition of landmarks. Systematic Zoology, 39(1):40-59, 1990. ISSN 00397989. doi: 10.2307/2992207. URL http://www.jstor. org/stable/2992207.

S. B. Shah and N. V. Sahinidis. Sas-pro: Simultaneous residue assignment and structure superposition for protein structure alignment. PloS one, 7 (5):-37493, 2012. doi: 10.1371/journal.pone.0037493.

Yosi Shibberu, Mark Brandt, and Allen Holder. Fundamentals of protein structure alignment. In Yi Pan, Jianxin Wang, and Min Li, editors, Algorithmic and AI Methods for Protein Bioinformatics, Wiley Book Series on Bioinformatics. Wiley, 2012.

Tsuyoshi Shimi, Tohru Kobayashi, Susumu Ito, and Koki Horikoshi. Protein Adaptation in Extremophiles, chapter Alkaline Adaptation of Proteins, pages 105-141. Nova Publishers, 2008.

Tsuyoshi Shirai, Kazuaki Igarashi, Tadahiro Ozawa, Hiroshi Hagihara, Tohru Kobayashi, Katsuya Ozaki, and Susumu Ito. Ancestral sequence evolutionary trace and crystal structure analyses of alkaline $\alpha$-amylase from bacillus sp. ksm-1378 to clarify the alkaline adaptation process of proteins. Proteins: Structure, Function, and Bioinformatics, 66(3):600-610, 2007. 
Tsuyoshi Shirai, Vo Si Hung, Katsuhito Morinaka, Tohru Kobayashi, and Susumu Ito. Crystal structure of gh13 $\alpha$-glucosidase gsj from one of the deepest sea bacteria. Proteins: Structure, Function, and Bioinformatics, 73(1):126-133, 2008. doi: 10.1002/prot.22044.

Dennis E. Slice. Geometric morphometrics. Annual Review of Anthropology, 36(1):261-281, 2007. doi: 10.1146/annurev.anthro.34. 081804.120613. URL http://www.annualreviews.org/doi/abs/10. 1146/annurev . anthro.34.081804.120613.

Birte Svensson and Stefan Janecek. Glycoside hydrolase family 13. available at URL http://www.cazypedia.org/, March 2013.

J. Thompson, D. Higgins, and T. Gibson. Clustal w: improving the sensitivity of progressive multiple sequence alignment through sequence weighting, position-specific gap penalties and weight matrix choice. Nucleic Acids Research, 22:4673-4680, 1994. doi: 10.1093/nar/22.22.4673.

Alexandra Tonkova. Bacterial cyclodextrin glucanotransferase. Enzyme and Microbial technology, 22(8):678-686, 1998. doi: 10.1016/S0141-0229(97) 00263-9.

Noreen von Cramon-Taubadel, Brenda C. Frazier, and Marta Mirazon Lahr. The problem of assessing landmark error in geometric morphometrics: theory, methods, and modifications. American journal of physical anthropology, 134(1):24-35, 2007. doi: 10.1002/ajpa.20616.

Inken Wohlers, Noël Malod-Dognin, Rumen Andonov, and Gunnar W. Klau. Csa: comprehensive comparison of pairwise protein structure alignments. Nucleic Acids Research, 40(W1):-303, 2012. doi: 10.1093/nar/ gks362. URL http://nar.oxfordjournals.org/content/40/W1/W303. abstract.

Peter E. Wright and H. Jane Dyson. Intrinsically unstructured proteins: reassessing the protein structure-function paradigm. Journal of molecular biology, 293(2):321-331, 1999. doi: 10.1006/jmbi.1999.3110.

Keizo Yamamoto, Hideo Miyake, Masami Kusunoki, and Shigeyoshi Osaki. Steric hindrance by 2 amino acid residues determines the substrate specificity of isomaltase from Saccharomyces cerevisiae. Journal of bioscience 
and bioengineering, 112(6):545-550, 2011. doi: 10.1016/j.jbiosc.2011.08. 016 .

Takehiro Yokota, Takashi Tonozuka, Yoichiro Shimura, Kazuhiro Ichikawa, Shigehiro Kamitori, and Yoshiyuki Sakano. Structures of thermoactinomyces vulgaris r-47 $\alpha$-amylase ii complexed with substrate analogues. Bioscience, biotechnology, and biochemistry, 65(3):619-626, 2001. doi: 10.1271/bbb.65.619.

M. L. Zelditch, D. L. Swiderski, H. D. Sheets, and W. L. Fink. Geometric morphometrics for biologists: A primer. Elsevier Academic Press, New York, 2004. 